УДК 612.393.1:636.081.113:581.149:341.321.1(470+571)

Для цитирования: Разводовский Ю.Е., Зотов П.Б. Продажа алкоголя и гендерный градиент ожидаемой продолжительности жизни в России. Сибирский вестник психиатрии и наркологии. 2018; 4 (101): 35-39. https://doi.org/10.26617/1810-31112018-4(101)-35-39

\title{
Продажа алкоголя и гендерный градиент ожидаемой продолжительности жизни в России
}

\section{Разводовский Ю.Е. ${ }^{1}$, Зотов П.Б. ${ }^{2}$}

${ }^{1}$ Гродненский государственный медищинский университет

Республика Беларусь, 230009, Гродно, ул. Горького, 80

${ }^{2}$ Тюменский государственный медииинский университет

Россия, 625023, Тюмень, Одесская улица, 54

\section{PEЗЮME}

Введение. Ожидаемая продолжительность жизни (ОПЖ) при рождении является индикатором качества жизни и здоровья населения. Гендерный градиент ОПЖ в России один из самых высоких в мире. Цель: изучение влияния структуры продажи алкоголя на динамику гендерного градиента ОПЖ в России. Методы. В сравнительном аспекте была проанализирована динамика продажи различных видов алкогольных напитков и гендерной разницы ОПЖ в России в период с 1970 г. по 2015 г. Статистическая обработка данных проводилась с помощью программного пакета «Statistica 12. StatSoft» в модуле «Анализ временных рядов». Результаты. Уровень продажи водки статистически значимо ассоциируется с гендерной разницей ОПЖ; рост уровня продажи водки на 1 литр приводит к росту гендерной разницы ОПЖ на 4,1\%. Выводы. Высокий уровень потребления крепких алкогольных напитков является одним из основных факторов высокого гендерного градиента ОПЖ, а изменения в уровне доступности водки являются главной причиной резких колебаний данного показателя в России.

Ключевые слова: продажа алкоголя, ожидаемая продолжительность жизни, гендерная разница, России, качество жизни и здоровья населения.

\section{ВВЕДЕНИЕ}

Ожидаемая продолжительность жизни при рождении является важным демографическим показателем и признанным индикатором качества жизни и здоровья населения $[1,2,3]$. Разница ОПЖ мужчин и женщин значительно варьирует в отдельных странах, при этом в странах Восточной Европы данный показатель существенно выше, чем в странах Западной Европы $[4,5]$. Гендерный градиент ОПЖ в России является одним из наиболее высоких во всём мире [6].

В предыдущих исследованиях показано, что одним из факторов высокого гендерного градиента ОПЖ в России является высокий уровень потребления алкоголя $[1,3,7,8]$. Хорошо известным фактом является то, что риск связанной с алкоголем смертности определяется не только общим уровнем потребления алкоголя, но и структурой потребления $[9,10,11,12]$. Высокий уровень смертности российских мужчин ассоциируется с распространенностью употребления больших доз крепких алкогольных напитков в течение короткого промежутка времени $[2,8,13]$. Данные предпосылки позволяют ожидать существования тесной связи между продажей водки и гендерным градиентом ОПЖ на популяционном уровне.

\section{ЦЕЛЬ ИССЛЕДОВАНИЯ}

Изучить влияние структуры продажи алкоголя на динамику гендерного градиента ожидаемой продолжительности жизни населения в России.

\section{МАТЕРИАЛ И МЕТОДЫ}

В сравнительном аспекте была проанализирована динамика продажи различных видов алкогольных напитков и динамика разницы ОПЖ женщин и мужчин в период с 1970 г. по 2015 г. Для оценки и удаления тренда из временного ряда использовался метод простых разностных операторов, суть которого заключается в переходе от исходного ряда к ряду разностей соседних значений ряда [14]. Метод авторегрессии - проинтегрированного скользящего среднего (АРПСС) использовался в настоящей работе с целью оценки степени связи между уровнем продажи различных видов алкогольных напитков (независимая переменная) и гендерной разницей ОПЖ (зависимая переменная). Статистическая обработка данных проводилась с помощью программного пакета «Statistica 12. StatSoft» в модуле «Анализ временных рядов».

РЕЗУЛЬТАТЫ

В период с 1970 г. по 2015 г. ОПЖ мужчин и женщин выросла соответственно на 1,8 (с 62,9 до $64,7)$ и 3,3 (73,0 до 76,3) года.

На рисунке 1 представлена динамика ОПЖ для обоих полов. Данный показатель демонстрировал тенденцию к снижению вплоть до начала 1980-х гг. (причем темпы снижения ОПЖ мужчин были более выражены); несколько вырос в 1982 г.; существенно вырос с 1984 г. по 1986 г.; снижался в период с 1987 г. по 1994 г., достигнув минимума за весь рассматриваемый период $(57,4$ и 71,1 года для мужчин и женщин соответственно); снова стал расти в период с 1995 г. по 1998 г.; снижался в период с 1999 г. по 2003 гг., после чего снова стал повышаться, достигнув самого высокого уровня за весь рассматриваемый период в 2014 г. (65,3 и 76,5 года для мужчин и женщин соответственно). 


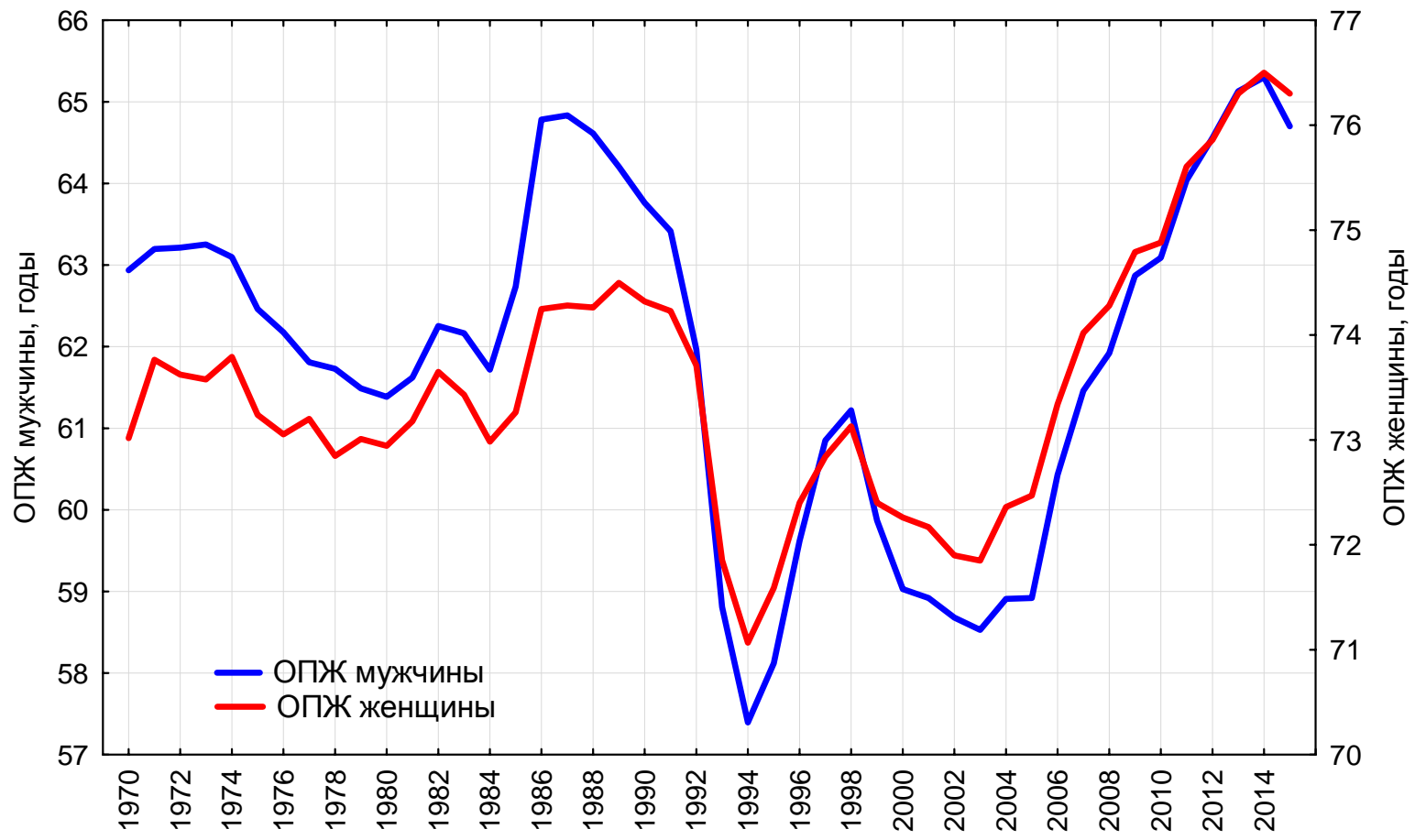

Р и с у н о к 1 . Динамика ожидаемой продолжительности жизни мужчин и женщин в России в период с 1970 г. по 2015 г.

Как показано на рисунке 2, гендерная разница ОПЖ в рассматриваемый период увеличилась на 1,5 (с 10,1 до 11,6) года. В целом динамика гендерного градиента ОПЖ оказалась аналогичной с динамикой ОПЖ.

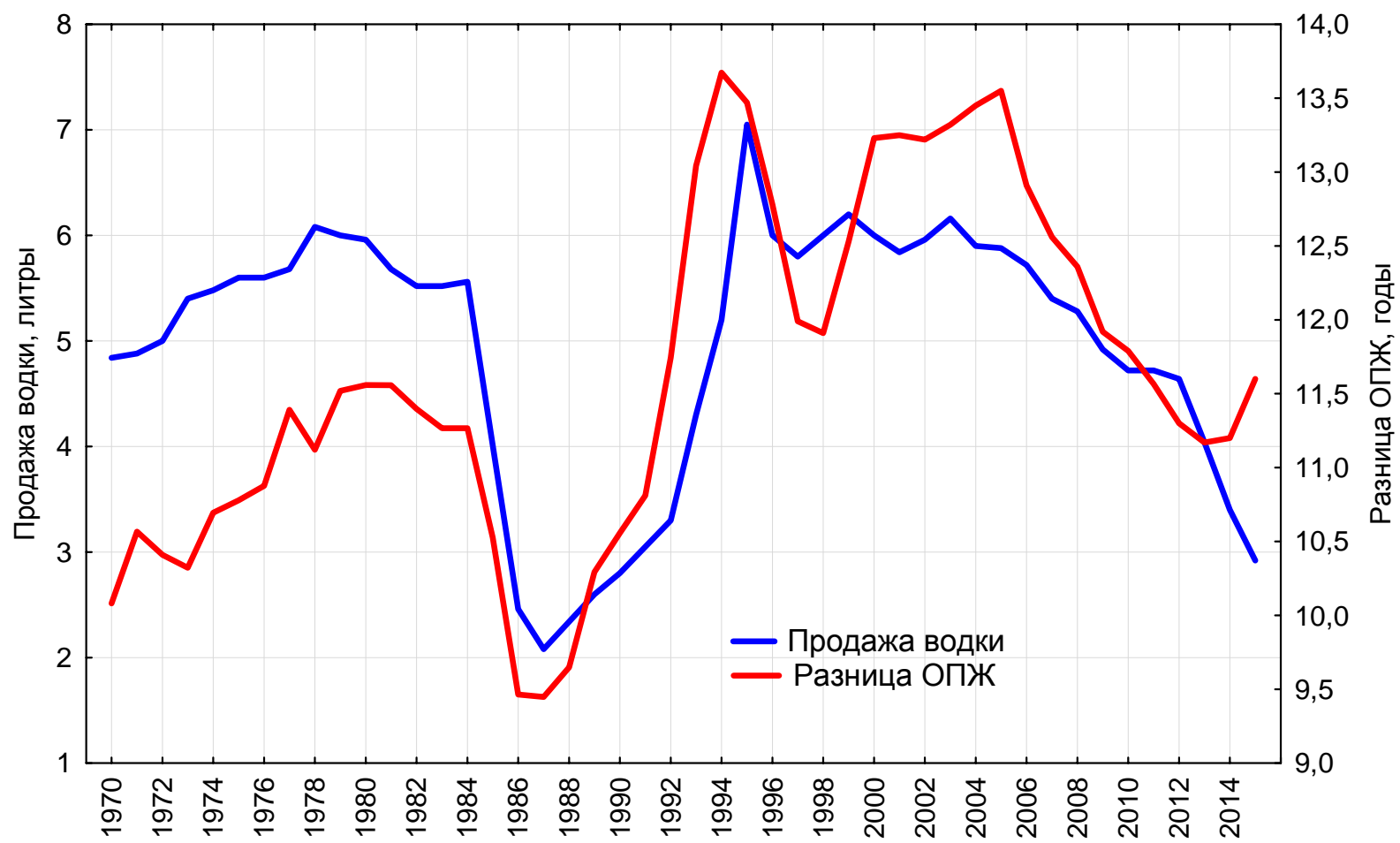

Р и с у н о к 2. Динамика гендерной разницы ожидаемой продолжительности жизни мужчин и женщин, а также уровня продажи водки в России с 1970 г. по 2015 г. 
В период с 1984 г. по 1986 г. данный показатель резко снизился, достигнув минимального значения за весь рассматриваемый период (9,5 года). В период с 1987 г. по 1994 г. гендерный градиент ОПЖ резко вырос, достигнув самого высокого уровня за весь рассматриваемый период (13,7 года). На протяжении последнего десятилетия рассматриваемого периода отмечалось значительное снижение уровня данного показателя. Резкие колебания гендерного градиента ОПЖ в рассматриваемый период были

обусловлены более выраженными колебаниями уровня ОПЖ мужчин.

Графические данные, представленные на рисунке 2, говорят о схожей динамике гендерного градиента ОПЖ и уровня продажи водки. После удаления тренда с помощью дифференцирования была оценена связь между временными рядами.

Кросс-корреляционный анализ преобразованных временных рядов показал существование тесной связи между уровнем продажи водки и гендерной разницей ОПЖ на нулевом лаге (табл. 1).

Т а б л и ц а 1

Связь между уровнем продажи различных видов алкогольных напитков и гендерной разницей ОПж. Результаты кросс-корреляционного анализа после удаления временного тренда

\begin{tabular}{|c|c|c|c|c|c|c|}
\hline \multirow{2}{*}{ Lag } & \multicolumn{2}{|c|}{ Продажа водки } & \multicolumn{2}{c|}{ Продажа вина } & \multicolumn{2}{c|}{ Продажа пива } \\
\cline { 2 - 7 } & $\mathrm{r}$ & $\mathrm{SE}$ & $\mathrm{r}$ & $\mathrm{SE}$ & $\mathrm{r}$ & $\mathrm{SE}$ \\
\hline-3 & 0,13 & 0,154 & 0,14 & 0,154 & 0,05 & 0,154 \\
\hline-2 & 0,26 & 0,153 & 0,29 & 0,153 & 0,01 & 0,153 \\
\hline-1 & 0,27 & 0,151 & 0,18 & 0,151 & 0,15 & 0,151 \\
\hline 0 & 0,49 & 0,149 & 0,04 & 0,149 & 0,24 & 0,149 \\
\hline 1 & 0,22 & 0,151 & 0,08 & 0,151 & 0,05 & 0,151 \\
\hline 2 & 0,08 & 0,152 & 0,11 & 0,152 & 0,18 & 0,152 \\
\hline 3 & 0,17 & 0,154 & 0,04 & 0,154 & 0,04 & 0,154 \\
\hline
\end{tabular}

Связь между уровнем продажи вина/пива и гендерной разницей ОПЖ также положительная, однако она статистически не значима. Поэтому для дальнейшего анализа использовались данные уровня продажи водки. Результаты анализа с использованием метода АРПСС выявили закономерность, согласно которой рост уровня продажи водки на 1 литр сопровождается ростом гендерной разницы ОПЖ на $4,1 \%$.

\section{ОБСУЖДЕНИЕ}

Результаты анализа временных серий, проведенных в рамках настоящего исследования, подтверждают рабочую гипотезу о существовании тесной связи между продажей водки и гендерной разницей ОПЖ на популяционном уровне. Резкие колебания гендерного градиента ОПЖ в рассматриваемый период были связаны с изменением уровня физической и экономической доступности водки. Так, снижение гендерного градиента ОПЖ в середине 1980-х гг., вероятнее всего, было обусловлено снижением доступности водки в период антиалкогольной кампании $[15,16]$. Увеличение доступности водки вследствие снижения государственного контроля за алкогольным рынком привело к резкому росту уровня связанной с алкоголем смертности среди мужчин в первой половине 90-х гг. прошлого века [17].

Резкий рост ОПЖ мужчин и женщин, так же как и снижение гендерного градиента ОПЖ, после 2005 г. является уникальным феноменом, который активно обсуждался специалистами в области общественного здоровья, однако так и не получил исчерпывающего объяснения [3, 18, 19]. Ранее было показано, что реальные темпы роста ОПЖ мужчин после 2005 г. значительно превосходили прогнозные темпы роста, полученные с помощью экстраполяции временного ряда, что позволило авторам предположить причастность к этому сценарию антиалкогольных законов, принятых в 2005 г., а также в последующие годы [3]. Ужесточение антиалкогольного законодательства, приведшее к снижению доступности водки, явилось интервенцией, существенно скорректировавшей динамику ОПЖ, которая стала значительно отличаться от тренда, предсказанного на основании предшествовавшей динамики временного ряда [3].

В заключении следует остановиться на методологических ограничениях данного исследования. В первую очередь это касается качества данных уровня продажи алкоголя, учитывая существование в России обширного теневого рынка крепкого алкоголя [20]. Кроме того, существенным ограничением данного исследования является игнорирование многочисленных переменных, помимо алкоголя, которые могли оказать влияние на уровень и динамику гендерного градиента ОПЖ. По всей видимости, проявлением влияния неучтенных переменных является рост гендерного градиента ОПЖ на фоне снижения уровня продажи алкогольной продукции в 2015 г.

\section{ВЫВОДЫ}

Представленные в настоящем исследовании данные говорят в пользу того, что высокий уровень потребления крепких алкогольных напитков является одним из основных факторов высокого гендерного градиента ОПЖ, а изменения в уровне доступности водки являются главной причиной резких колебаний данного показателя в России. Следовательно, государственная алкогольная политика, направленная на ограничение доступности водки, является важным инструментом снижения гендерного градиента ОПЖ. 


\section{КОНФЛИКТ ИНТЕРЕСОВ}

Авторы заявляют об отсутствии конфликта интересов в связи с публикацией данной статьи.

\section{ИСТОЧНИК ФИНАНСИРОВАНИЯ}

Авторы заявляют об отсутствии финансирования при проведении исследования.

\section{СООТВЕТСТВИЕ ПРИНЦИПАМ ЭТИКИ}

Исследование проведено с соблюдением норм современной биомедицинской этики и этических стандартов, разработанных в соответствии с Хельсинской декларацией ВМА.

\section{ЛИТЕРАТУРА}

1. Коссова Т., Коссова Е., Шелунцова М. Влияние потребления алкоголя на смертность и ожидаемую продолжительность жизни в регионах России. Экономическая политика. 2017; 12 (1): 58-83.

2. Иванова А.Е., Семенова В.Г., Гаврилова Н.С., Евдокушкина Г.П., Гаврилов Л.А. Российская смертность в 1965 2002 гг.: основные проблемы и резервы снижения. Общественное здоровье и профилактика заболеваний. 2004; 1: 20-30.

3. Разводовский Ю.Е., Смирнов В.Ю. Прогнозирование ожидаемой продолжительности жизни с помощью анализа временных серий. Собриология. 2016; 1: 32-36.

4. Barret-Connor E. Gender differences and disparities in allcause and coronary heart disease mortality: epidemiological aspect. Best Pract Res Clin Endocrinol Metab. 2013; 27 (4): 481-500.

5. Разводовский Ю.Е., Смирнов В.Ю. Прогнозирование уровня общей смертности в Беларуси. Научный форум. Сибирь. 2017; 3 (2): 42-45.

6. Van Oyen H., Nusselder W., Jagger C., Kolip P., Cambois E., Robine J.M. Gender differences in healthy life years within the EU: an exploration of the "health-survival" paradox. Int J Public Health. 2013; 58: 143-155.

7. Разводовский Ю.Е. Алкоголь и суицид в странах Восточ- ной Европы. Суииидология. 2014; 5 (3): 18-27.

8. Moskalewicz J., Razvodovsky Y.E., Wieczorek P. East-West disparities in alcohol-related harm. Alcoholism and Drug Addiction. 2016; 29: 209-222.

9. Razvodovsky Y.E. Beverage-specific alcohol sale and cardiovascular mortality in Russia. Journal of Environmental and Public Health. 2010:Article ID:253853

10. Разводовский Ю.Е. Алкогольные отравления и гендерный градиент ожидаемой продолжительности жизни в Беларуси. Девиантология. 2017; 1 (1): 19-24.

11. Немцов А.В., Шелыгин К.В. Самоубийства и потребление алкоголя в России, 1956-2013 гг. Суицидология. 2016; 7 (3): $3-12$.

12. Родяшин Е.В., Зотов П.Б., Габсалямов И.Н., Уманский М.С. Алкоголь среди факторов смертности от внешних причин. Суицидология. 2010; 1:21-23.

13. Котельникова 3.В. Взаимосвязь практик потребления алкоголя с социальной структурой современной России. Социологические исследования. 2015; 4: 105-112.

14. Box G.E.P., Jenkins G.M. Time series analysis: forecasting and control. GEP. Box. London: Holden-Day Inc., 1976.

15. Razvodovsky Y.E. Estimation of alcohol attribute able fraction of mortality in Russia. Alcoholism. 2012; 48 (1): 13-22.

16. Немцов А.В., Разводовский Ю.Е. Оценка уровня потребления алкоголя в России: обзор литературы. Собриология. $2017 ; 1: 78-88$.

17. Razvodovsky Y.E. Beverage specific alcohol sale and mortality in Russia. Alcoholism. 2010; 46: 63-75.

18. Разводовский Ю.Е., Немцов А.В. Алкогольная составляющая снижения смертности в России после 2003 г. Вопросы наркологии. 2016; 3: 63-70.

19. Nemtsov A.V., Razvodovsky Y.E. Russian alcohol policy in false mirror. Alcohol \& Alcoholism. 2016; 4: 21.

20. Razvodovsky Y.E. Unrecorded alcohol consumption: quantitative methods of estimation. Alcoholism. 2010; 46 (1): 15-24.

Поступила в редакцию 4.06.2018 Утверждена к печати 6.11.2018

Разводовский Юрий Евгеньевич - кандидат медицинских наук, старший научный сотрудник научно-исследовательской лаборатории. Зотов Павел Борисович - доктор медицинских наук, профессор (SPIN-код: 5702-4899, ORCID iD: 0000-0002-1826-486X, Researcher ID: U2807-2017), заведующий кафедрой онкологии.

УДК 612.393.1:636.081.113:581.149:341.321.1(470+571)

For citation: Razvodovsky Yu.E., Zotov P.B. Alcohol sales and gender difference in life expectancy in Russia. Siberian Herald of Psychiatry and Addiction Psychiatry. 2018; 4 (101): 35-39. https://doi.org/10.26617/1810-3111-2018-4(101)-35-39

\section{Alcohol sales and gender difference in life expectancy in Russia Razvodovsky Yu.E. ${ }^{1}$, Zotov P.B. ${ }^{2}$ \\ ${ }^{1}$ Grodno State Medical University Gorky Street 80, 230009, Grodno, Republic of Belarus \\ ${ }^{2}$ Tyumen State Medical University Odesskaya Street 54, 625023, Tyumen, Russian Federation}

\section{ABSTRACT}

Background: Life expectancy at birth (LEB) is considered to be an important characteristic for quality of life. The difference in life expectancy at birth between males and females in Russia is unprecedently high compared with developed countries. Objective: The aim of the current study was to examine the aggregate-level relationship between the consumption of different alcoholic beverages and gender difference in LEB in Russia between 1970 and 2015. Method: To estimate the relation between these variables across the study period a time series analysis was performed using the statistical package "Statistica 12. Stat Soft". Results: The results of analysis indicate that vodka sales are closely linked with gender difference in LEB in Russia: an additional liter of vodka sales per capita was estimated to increase in the difference between male and female LEB by $4.1 \%$. Conclusions: This piece of evidence provides support for the hypothesis that high level of vodka consumption in conjunction with binge drinking pattern may be a major reason for the high gender difference in LEB and its dramatic fluctuations in Russia during the last few decades. 


\section{Keywords: alcohol sales, life expectancy, gender difference, Russia, quality of life and health of the population.}

\section{REFERENCES}

1. Kossova T., Kossova E., Sheluntsova M. Vliyanie potrebleniya alkogolya na smertnost' i ozhidaemuyu prodolzhitel'nost' zhizni $v$ regionah Rossii [The effect of alcohol consumption on mortality and life expectancy in the regions of Russia]. Ekonomicheskaya politika - Economic Policy. 2017; 12 (1): 58-83 (in Russian).

2. Ivanova A.E., Semenova V.G., Gavrilova N.S., Evdokushkina G.P., Gavrilov L.A. Rossijskaya smertnost' v 1965-2002 gg.: osnovnye problemy i rezervy snizheniya [Russian mortality in 1965-2002.: main problems and reserves for reduction]. $O b$ shchestvennoe zdorov'e $i$ profilaktika zabolevanij - Public Health and Disease Prevention. 2004; 1: 20-30 (in Russian).

3. Razvodovsky Yu.E., Smirnov V.Yu. Prognozirovanie ozhidaemoj prodolzhitel'nosti zhizni s pomoshch'yu analiza vremennyh serij [Prediction of life expectancy using the analysis of time series]. Sobriologiya - Sobriology. 2016; 1: 32-36 (in Russian).

4. Barret-Connor E. Gender differences and disparities in all-cause and coronary heart disease mortality: epidemiological aspect. Best Pract Res Clin Endocrinol Metab. 2013; 27(4): 481-500.

5. Razvodovskyj Yu.E., Smirnov V.Yu. Prognozirovanie urovnya obshchej smertnosti v Belarusi [Prediction of total mortality rate in Belarus]. Nauchnyj forum. Sibir' - Scientific Forum. Siberia. 2017; 3 (2): 42-45 (in Russian).

6. Van Oyen H., Nusselder W., Jagger C., Kolip P., Cambois E., Robine J.M. Gender differences in healthy life years within the EU: an exploration of the "health-survival" paradox. Int J Public Health. 2013; 58: 143-155.

7. Razvodovsky Yu.E. Alkogol' i suicid v stranah Vostochnoj Evropy [Alcohol and suicide in Eastern Europe.]. Suicidologiya - Suicidology. 2014; 5 (3): 18-27 (in Russian). .

8. Moskalewicz J., Razvodovsky Y.E., Wieczorek P. East-West disparities in alcohol-related harm. Alcoholism and Drug Addiction. 2016; 29: 209-222.

9. Razvodovsky Y.E. Beverage-specific alcohol sale and cardiovascular mortality in Russia. Journal of Environmental and Public Health. 2010:Article ID:253853
10. Razvodovsky Yu.E. Alkogol'nye otravleniya i gendernyj gradient ozhidaemoj prodolzhitel'nosti zhizni v Belarusi [Alcohol poisoning and gender gradient of life expectancy in Belaru]. Deviantologiya-Deviantology. 2017; 1 (1): 19-24 (in Russian).

11. Nemtsov A.V., Shelygin K.V. Samoubijstva i potreblenie alkogolya v Rossii, 1956-2013 gg. [Suicides and alcohol consumption in Russia, 1956-201] Suicidologiya - Suicidology. 2016; 7 (3): 3-12 (in Russian).

12. Rodyashin E.V., Zotov P.B., Gabsalyamov I.N., Umansky M.S. Alkogol' sredi faktorov smertnosti ot vneshnih prichin [Alcohol is among the factors of mortality from external causes]. Suicidologiya - Suicidology. 2010; 1: 21-23 (in Russian).

13. Kotel'nikova Z.V. Vzaimosvyaz' praktik potrebleniya alkogolya s social'noj strukturoj sovremennoj Rossii [The relationship of practices of alcohol consumption with the social structure of modern Russia.]. Sociologicheskie issledovaniya -Sociological Research. 2015; 4: 105-112 (in Russian).

14. Box G.E.P., Jenkins G.M. Time series analysis: forecasting and control. GEP. Box. London: Holden-Day Inc., 1976.

15. Razvodovsky Y.E. Estimation of alcohol attribute able fraction of mortality in Russia. Alcoholism. 2012; 48 (1): 13-22.

16. Nemtsov A.V., Razvodovsky Yu.E. Ocenka urovnya potrebleniya alkogolya v Rossii: obzor literatury [Assessment of the level of alcohol consumption in Russia: a literature review]. Sobriologiya - Sobriology. 2017; 1: 78-88 (in Russian).

17. Razvodovsky Y.E. Beverage specific alcohol sale and mortality in Russia. Alcoholism. 2010; 46: 63-75.

18. Razvodovsky Yu.E., Nemtsov A.V. Alkogol'naya sostavlyayushchaya snizheniya smertnosti v Rossii posle 2003 g. [Alcoholic component of mortality decline in Russia after 2003] Voprosy narkologii - Journal of Addiction Problems. 2016; 3: 63-70 (in Russian).

19. Nemtsov A.V., Razvodovsky Y.E. Russian alcohol policy in false mirror. Alcohol \& Alcoholism. 2016; 4: 21.

20. Razvodovsky Y.E. Unrecorded alcohol consumption: quantitative methods of estimation. Alcoholism. 2010; 46 (1): 15-24.

Received June 4.2018 Accepted November 6.2018

Razvodovsky Yury E., PhD, senior researcher of the Research Laboratory, Grodno State Medical University, Grodno, Republic of Belarus. Zotov Pavel B., MD, Prof., Head of the Department of Oncology, Tyumen State Medical University, Tyumen, Russian Federation 